\section{RMD Open}

Rheumatic \&

Musculoskeletal Diseases

\title{
A multicentre, randomised, controlled, open-label pilot study on the feasibility of discontinuation of adalimumab in established patients with rheumatoid arthritis in stable clinical remission
}

To cite: Chatzidionysiou $\mathrm{K}$, Turesson C, Teleman A, et al. A multicentre, randomised, controlled, open-label pilot study on the feasibility of discontinuation of adalimumab in established patients with rheumatoid arthritis in stable clinical remission. RMD Open 2016;2:e000133. doi:10.1136/rmdopen-2015000133

- Prepublication history for this paper is available online To view these files please visit the journal online (http://dx.doi.org/10.1136/ rmdopen-2015-000133).

Received 28 May 2015 Revised 24 November 2015 Accepted 26 November 2015

\section{CrossMark}

For numbered affiliations see end of article.

Correspondence to Dr Katerina Chatzidionysiou; aikaterini.chatzidionysiou@ karolinska.se

\section{ABSTRACT}

Objectives: Treatment with tumour necrosis factor (TNF) blockers, once started as therapy for rheumatoid arthritis (RA), is usually continued indefinitely. The aim of this trial was to assess the possibility of discontinuing treatment with adalimumab (ADA) while maintaining remission in patients with RA with established disease in stable remission on combination therapy with ADA and methotrexate (MTX).

Methods: In a randomised, controlled, open-label pilot study of patients with RA in stable remission treated with $\mathrm{ADA}+\mathrm{MTX}$, patients were randomised in a 1:1 ratio to continue with ADA plus MTX (arm AM) or MTX monotherapy (arm M) for 52 weeks. Flare was defined as Disease Activity Score (DAS28) $\geq 2.6$ or a change in DAS28 ( $\triangle \mathrm{DAS} 28)$ of $>1.2$ from baseline at any time. Patients in arm $M$ with a flare restarted ADA. The primary end point was the proportion of patients in remission at week 28.

Results: 31 patients were enrolled in the study and randomised to arm AM $(n=16)$ or arm $M(n=15)$. At 28 weeks, $15 / 16$ patients (94\%) and 5/15 patients $(33 \%)$ in arms $A M$ and $M$, respectively, were in remission ( $p=0.001)$. During the first 28 weeks, $50 \%$ $(8 / 16)$ in the AM arm and $80 \%(12 / 15)$ in the $M$ arm had a flare $(p=0.08)$. The number of patients in the AM and $M$ arms with $\geq 1 \Delta \mathrm{DAS} 28>1.2$ during the first 28 weeks was $1 / 16(6 \%)$ and $8 / 15(53 \%)$, respectively $(p=0.005)$.

Conclusions: In this study, remission was rarely maintained in patients with long-standing disease who discontinued ADA. Discontinuation may be feasible in only a minority of patients with established RA in stable clinical remission.

Trial registration number: NCT00808509.

\section{INTRODUCTION}

The field of rheumatoid arthritis (RA) and the treatment of the disease have changed

\section{Key messages}

What is already known about this subject?

- Information regarding the feasibility of discontinuing antitumour necrosis factor therapy in patients with established rheumatoid arthritis (RA) who have obtained remission is limited.

What does this study add?

- The ADMIRE trial showed that remission was rarely maintained in patients with established RA who discontinued adalimumab.

How might this impact on clinical practice?

- In clinical practice, discontinuation of adalimumab may be possible in established RA, but in a minority of patients.

- All patients experiencing disease flare who restarted treatment with adalimumab achieved remission without any unexpected safety signals.

dramatically during the past decade. Better understanding of the pathophysiology and the underlying immunological mechanisms of RA has led to tighter disease control, earlier treatment and the emergence of a new class of drugs, biological diseasemodifying antirheumatic drugs (DMARDs). Tumour necrosis factor (TNF) inhibitors were the first biologics to be approved for the treatment of severe RA. Adalimumab (ADA) is a recombinant human immunoglobulin (IgG1) monoclonal antibody that binds with high affinity and specificity to TNF. $^{1}$ The efficacy of ADA in the treatment of RA and its acceptable safety profile have been demonstrated in large randomised, controlled clinical trials. ${ }^{23}$ 
The goal of treatment today is remission: clinical, functional and radiographic. A further step is a sustained remission state without the need for continuous treatment with a biological agent-the achievement of a 'biological-free' remission. If remission could be sustained even after the cessation of anti-TNF therapy, this would have vast clinical (regarding long-term safety) as well as economic implications. Treatment with TNF blockers, once started as therapy for RA, is usually continued indefinitely. This is mainly due to the fact that information regarding the feasibility of discontinuing anti-TNF therapy in patients with RA who have obtained remission is limited. In the ATTRACT study, patients with long-standing RA receiving treatment with infliximab were followed; in 17 patients, treatment was discontinued after 2 years. ${ }^{4}$ All 17 patients experienced disease flare after discontinuing infliximab. In contrast, in a study of patients with early RA, 70\% of those initially treated with infliximab were able to discontinue TNF inhibitor therapy while remaining in remission. ${ }^{5}$ In the RRR study by Tanaka et $a l,{ }^{6}$ of 102 patients, $56(55 \%)$ maintained low disease activity (Disease Activity Score based on 28 joints (DAS28) <3.2) and $44(43 \%)$ fulfilled the criteria for clinical remission (DAS28 <2.6) 1 year after discontinuation of infliximab. The mean disease duration in this study was 6 years, suggesting that discontinuation of TNF inhibitors may be feasible not only in patients with early RA, but also in those with established and long-standing disease.

Apart from the duration of RA, other factors can influence the chance of biological-free remission, such as the time from disease diagnosis to the introduction of anti-TNF therapy. In the BeST study, which was an early-RA study, it was shown that significantly more patients who received initial combination therapy with infliximab and methotrexate (MTX) were able to discontinue infliximab compared with those having delayed introduction of a biological agent (56\% vs $29 \%$; $\mathrm{p}=0.008) .{ }^{7}$ In the OPTIMA trial, patients with early RA who achieved stable low disease activity with treatment that included ADA plus MTX, and subsequently discontinued treatment with ADA, mostly maintained their treatment response. ${ }^{8}$ The discontinuation of ADA may be feasible even in patients with more established RA, but this is so mainly in patients in deep remission, as noted in the HONOR study. ${ }^{9}$ In the PRIZE study, however, DMARD-naïve patients with early RA who achieved remission while receiving full-dose etanercept in combination with MTX, continuing combination therapy at a reduced dose, resulted in better disease control than those switching to MTX alone or placebo. ${ }^{10}$ In the DOSERA study, patients with established RA who had achieved stable low disease activity on etanercept in combination with MTX, continuing both, found that therapy superior to MTX alone. Reduced dose etanercept was also more effective than MTX alone in maintaining a favourable response. ${ }^{11}$ The PRESERVE trial yielded similar results. ${ }^{12}$ Finally, in the
CERTAIN trial, remission was maintained in a minority of patients after withdrawal of the TNF inhibitor (certolizumab pegol). ${ }^{13}$

Taken together, much of the available data on the discontinuation of TNF inhibitors come from studies of patients with early RA enrolled in double-blind clinical trials. There is a lack of studies of patients with longstanding disease seen in clinical practice, in particular on discontinuation of ADA.

The aim of this pilot study was to assess the possibility of discontinuing treatment with ADA while maintaining remission in patients with established disease in stable clinical remission on combination therapy with ADA plus MTX.

\section{METHODS}

\section{Study design}

This was a multicentre, randomised, controlled, openlabel, pilot study. The main inclusion criteria were: age $\geq 18$ years; diagnosis of RA based on the 1987 revised American College of Rheumatology (ACR) classification criteria, ${ }^{14}$ positive rheumatoid factor (RF) or at least one erosion on the radiograph of hands or feet; treatment with $\mathrm{ADA}$ in the approved dose of $40 \mathrm{mg}$ every other week for at least 6 months; concomitant treatment with MTX at a dose of at least $10 \mathrm{mg} /$ week for a minimum of 6 months (stable dose for a minimum of 2 months); stable remission according to the DAS28 (DAS28 <2.6), ${ }^{15}$ for at least 3 months based on assessments at study entry=baseline and on at least one more occasion 3-6 months prior to baseline, documented in patient record or registry. Concomitant corticosteroids were allowed if the dose was $10 \mathrm{mg} /$ day or less (prednisolone or equivalent) and had been stable for at least 3 months at baseline.

Patients fulfilling the inclusion criteria were randomised in a 1:1 ratio to arm AM (continue with ADA and MTX) or to arm M (discontinue ADA and continue with MTX monotherapy) for 52 weeks (figure 1). Any patient experiencing disease 'flare' at any visit could continue in the rescue arm, where ADA would be reinstituted. Disease flare was defined as DAS28 $\geq 2.6$ or a change in DAS28 ( $\triangle$ DAS28) $>1.2$ from baseline at any time. After week 52, an observational extension phase was initiated, in which patients were treated at the discretion of the investigator for an additional period of 52-104 weeks.

The study was conducted in accordance with the trial protocol, International Conference on Harmonisation (ICH) guidelines, applicable regulations and guidelines governing clinical study conduct and the ethical principles that have their origin in the Declaration of Helsinki. The study was registered at Clinical Trials.gov (NCT00808509).

\section{End points}

The primary end point of this trial was the proportion of patients in remission (according to DAS28 score) at week 28. Secondary end points included the incidence of disease flare and the evolution of physical function 
Figure 1 Study design. *Flare, DAS28 $\geq 2.6$ or a change in DAS28 ( $\triangle$ DAS28) $>1.2$ from baseline at any time. ADA, adalimumab; eow, every other week; MTX, methotrexate.

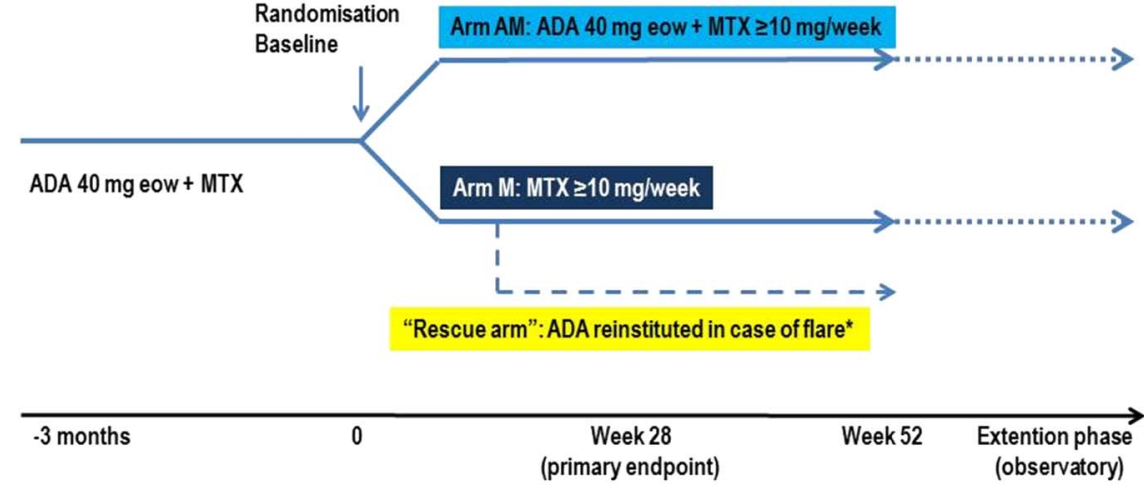

(assessed using the Swedish version of Health Assessment Questionnaire $\left.(\mathrm{HAQ})^{16}\right)$. Assessments of disease activity and physical function were performed every 4 weeks according to the protocol in both groups.

Further exploratory analyses were performed and included the following end points: (1) Incidence of disease flare (DAS28 $\geq 2.6$ or a $\Delta$ DAS28 $>1.2$ from baseline at any time); (2) Incidence of at least one DAS28 $\geq 2.6$ from baseline to week 28; (3) Incidence of at least one $\triangle$ DAS28 >1.2 from baseline to week 28; (4) Proportion of patients with at least one of the following from baseline to week 28: $\triangle$ DAS28 $>0.6$, DAS28 $\geq 2.6$ AND $\triangle$ DAS28 $>1.2$, DAS28 $\geq 2.6$ AND $\triangle$ DAS28 $>0.6$; (5) Proportion of patients in DAS28 remission at week 52; (6) Flare-free survival during the first 28 weeks; (7) Change in functional status (assessed by HAQ) at week 28; (8) Change in radiological status (analysis of radiographic data at week 52).

In addition, we applied the European League Against Rheumatism (EULAR)/ACR Boolean remission criteria $^{17}$ to the two arms and assessed the frequency of remission. Remission was defined according to the following criteria: swollen joint count $\leq 1$, tender joint count $\leq 1$, patient global assessment $\leq 1$ (on a $0-10$ scale) and erythrocyte sedimentation rate $\leq 20 \mathrm{~mm} / \mathrm{h}^{18} 19$

\section{Analysis of radiographic data and safety data}

Radiographs of hands (posteroanterior view) and feet (anteroposterior view) were performed at baseline

Figure 2 Disposition of patients through the study. *One patient was later excluded from each arm, one in the $\mathrm{M}$ arm due to a major protocol violation at week 8 and one in the AM arm who did not fulfil the inclusion criteria. Arm AM: patients treated with adalimumab and methotrexate; Arm M: patients treated with methotrexate only. (unless a comparable radiograph had been obtained within 3 months from baseline), at week 52 and at week 104 through week 156. The Sharp/van der Heijde 2021 (SvH) scoring method was used to assess radiographic progression. Adverse events were assessed at every visit throughout the study.

\section{Statistical analysis}

Proportions were compared between the two arms, using the Fisher exact test. Continuous variables were presented as median (IQR) and were compared using the non-parametric Mann-Whitney U test. The Wilcoxon signed rank test was used as non-parametric paired test to compare the change in HAQ and SvH score. Kaplan-Meier curves representing patients free of disease flare were compared by log-rank test. Analyses were performed using the intent-to-treat population. Non-responder imputation (ie, 'flare' imputed) was performed for patients with no available DAS28 score at week 28 (this included most patients who had a flare in the $\mathrm{M}$ arm and who restarted treatment with ADA).

\section{RESULTS}

\section{Patient population}

The disposition of patients through the study is shown in figure 2. From a total of 237 screened patients, only 33 (14\%) were enrolled in the study. A significant number

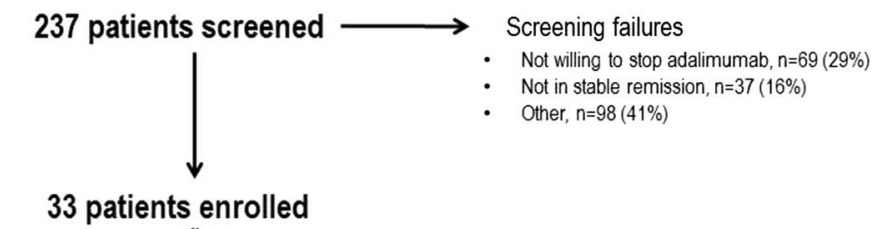


of patients (29\%) were not willing to stop ADA therapy; $16 \%$ of patients did not fulfil the criteria for stable remission; $12 \%$ were on ADA monotherapy or had a lower dose of MTX than $10 \mathrm{mg} /$ week; and $5 \%$ had a dose of ADA other than $40 \mathrm{mg}$, every other week. The remaining $41 \%$ of patients screened could not be enrolled for other reasons. Of the 33 patients enrolled, 17 were randomised to arm $\mathrm{AM}$ and 16 were randomised to arm M. One patient was later excluded from each arm-one in the $\mathrm{M}$ arm due to a major protocol violation at week 8 and one in the AM arm who did not fulfil the inclusion criteria. Patient baseline characteristics from the time of randomisation are shown in table 1 . No statistically significant difference was found between the arms regarding baseline characteristics except for age $(p=0.02)$. Three patients had low dose concomitant Prednisolone (two patients had $2.5 \mathrm{mg}$ /day and one patient had $5 \mathrm{mg} /$ day), all in the AM arm.

\section{Primary, secondary and exploratory end points}

At week 28, 15 of 16 patients in the AM arm and 5 of 15 patients in the $M$ arm were in remission $(p=0.001$; figure $3 \mathrm{~A}$ ). Two patients in the $\mathrm{M}$ arm who had disease flare did not want to restart ADA treatment. The proportion of patients with at least one disease flare (defined as at least one DAS28 $\geq 2.6$ or $\triangle$ DAS28 $>1.2$ ) during the first 28 weeks was $50 \%(8 / 16)$ in the AM arm and $80 \%$ (12/ $15)$ in the $M$ arm ( $p=0.08$; figure $3 B$ ). Analysis on the subgroups of patients with disease flare showed a significant difference in the proportion of patients with at least one $\triangle \mathrm{DAS} 28>1.2$ (figure 3D), but no difference in the proportion of patients with at least one DAS28 $\geq 2.6$ (figure 3C). When different definitions of disease flare were tested (an exploratory analysis as described in the Methods section), the difference between the AM and the $\mathrm{M}$ arm became clearer (figures $3 \mathrm{E}-\mathrm{G}$ ). Survival curves suggested higher flare-free survival over time in patients randomised to continue treatment with $\mathrm{ADA}$, although the difference did not reach statistical significance ( $p=0.07$; figure 4 ).

Approximately half of the patients in the two arms fulfilled the EULAR/ACR remission criteria at baseline (7/ 16 patients in arm AM and 9/15 patients in arm M). By the end of 28 weeks, 2 of 16 patients in arm AM and 1 of 15 patients in arm M fulfilled these criteria (no statistically significant difference).

During the study period, patients who experienced a disease flare had longer disease duration and started treatment with ADA later than those who did not experience a disease flare, in the $\mathrm{M}$ and $\mathrm{AM}$ arms (table 2). There was also a tendency for a lower baseline DAS28 in patients who did not experience a disease flare.

At week 52, 81\% of patients in arm AM (13/16) and $13 \%$ of patients in arm $\mathrm{M}(2 / 15)$ were in remission, while the rest of the patients in each arm were nonresponders after imputation. At the observatory visit (weeks 104-152), three of the patients originally randomised to the M arm had not restarted ADA, and two of these patients were in remission.

\section{Functional and radiographic status}

In arm AM, the median (IQR) HAQ was $0.13(0-0.7)$ at baseline and $0.32(0-0.7)$ at week 28 (Wilcoxon signed rank test, $\mathrm{p}=0.8$ ). In arm $\mathrm{M}$, the mean (SD) HAQ was $0.38(0.1-0.6)$ at baseline and $0.5(0.1-0.8)$ at week 28 $(\mathrm{p}=0.4)$. Median $\triangle \mathrm{HAQ}$ from baseline to week 28 was 0 $(0-0)$ in arm $\mathrm{AM}$ and $0(-0.12$ to 0.13$)$ in arm $\mathrm{M}$ $(\mathrm{p}=0.6)$. The proportion of patients with at least one

Table 1 Baseline characteristics in the two treatment arms

\begin{tabular}{|c|c|c|c|c|}
\hline & $\begin{array}{l}\text { AM Arm } \\
(n=16)\end{array}$ & $\begin{array}{l}\text { M Arm } \\
(n=15)\end{array}$ & $\begin{array}{l}\text { p Value } \\
\text { (AM vs M } \\
\text { arm) }\end{array}$ & $\begin{array}{l}\text { All patients } \\
(\mathrm{N}=31)\end{array}$ \\
\hline Age (years, median (IQR)) & $56(38.8-62)$ & $64(59-66)$ & 0.02 & $61(53-65)$ \\
\hline Sex (male/female (female)) & $10 / 16(62.5 \%)$ & $10 / 15(66.7 \%)$ & 0.56 & $20 / 31(64.5 \%)$ \\
\hline Disease duration (years, median (IQR)) & $7.6(4.0-12.1)$ & $10.4(5.2-19.2)$ & 0.45 & $8.0(4.8-16.2)$ \\
\hline Time (months) on ADA at baseline (median (IQR)) & $\begin{array}{l}26.5(12.5- \\
51.2)\end{array}$ & $\begin{array}{l}43.3(11.7- \\
51.5)\end{array}$ & 0.19 & $29.1(12.3-51.2)$ \\
\hline $\begin{array}{l}\text { Time from RA diagnosis to ADA start (years, median } \\
\text { (IQR)) }\end{array}$ & $3.2(2.2-9.5)$ & $6.5(4.1-15.1)$ & 0.63 & $4.8(2.8-10.4)$ \\
\hline RF (positive) & $11 / 16(68.8 \%)$ & $11 / 12(91.7 \%)$ & 0.16 & $22 / 28(78.6 \%)$ \\
\hline Anti-CCP (positive) & $5 / 10(50 \%)$ & $8 / 9(88.9 \%)$ & 0.09 & $13 / 19(68.4 \%)$ \\
\hline Number of previous DMARDs (median (IQR)) & $2(1-3)$ & $2(2-3)$ & 0.99 & $2(1-3)$ \\
\hline Number of previous biologics (median (IQR)) & $0(0-1)$ & $0(0-1)$ & 0.83 & $0(0-1)$ \\
\hline Baseline DAS28 (median (IQR)) & $2.13(1.6-2.4)$ & $1.69(1.5-2.37)$ & 0.45 & $1.9(1.55-2.39)$ \\
\hline Baseline HAQ (median (IQR)) & $0.13(0-0.72)$ & $\begin{array}{l}0.38(0.13- \\
0.63)\end{array}$ & 0.47 & $0.38(0-0.63)$ \\
\hline Concomitant MTX dose (median (IQR)) & $20(15-20)$ & $20(10-20)$ & 0.55 & $20(15-20)$ \\
\hline
\end{tabular}

ADA, adalimumab; anti-CCP, anticyclic citrullinated peptide antibodies; DAS28, Disease Activity Score based on 28 joints; DMARD, disease-modifying antirheumatic drugs; HAQ, Health Assessment Questionnaire; RA, rheumatoid arthritis; RF, rheumatoid factor. 


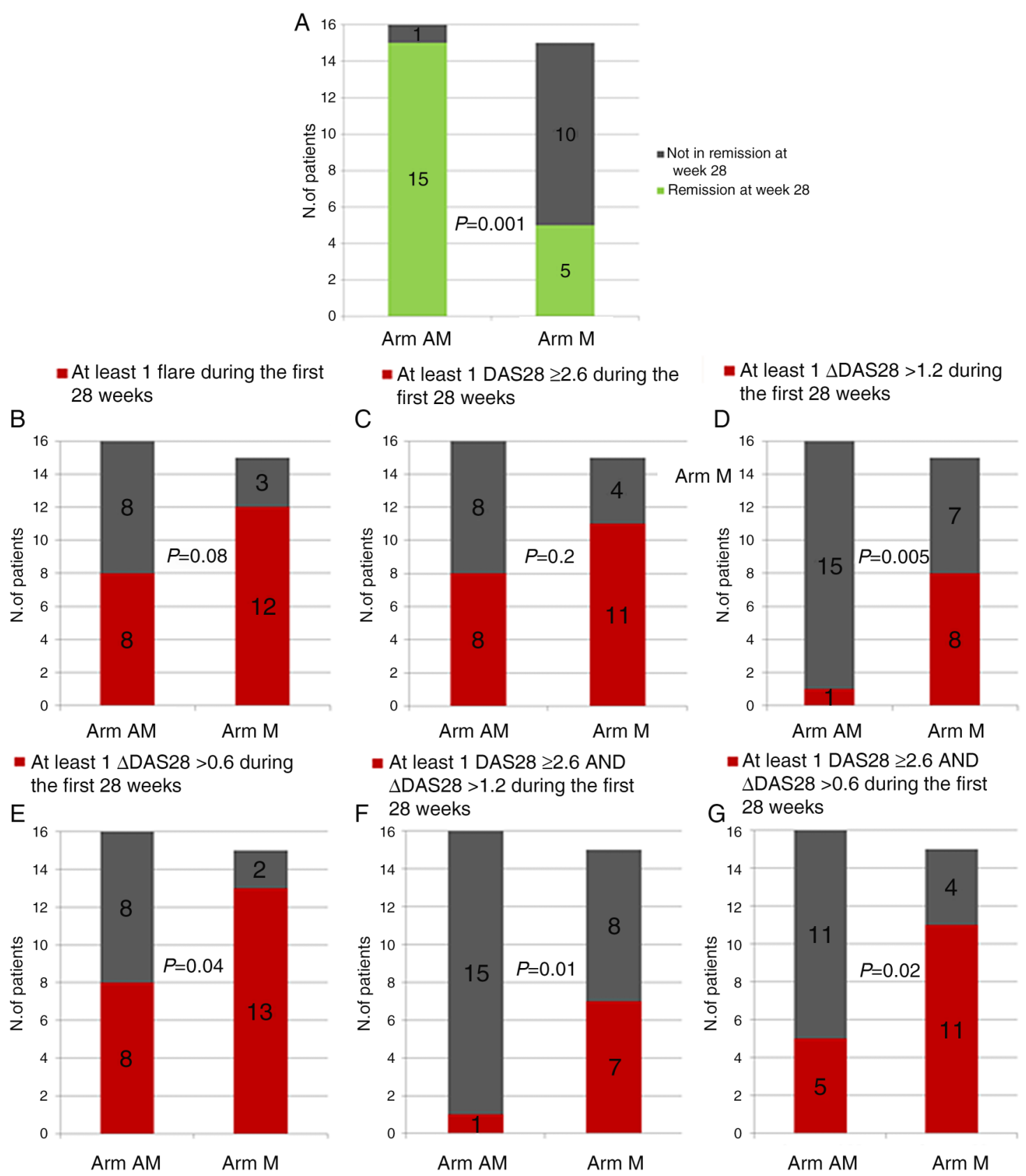

Figure 3 Primary $(A)$ and secondary $(B-G)$ end points. Arm AM: patients treated with adalimumab and methotrexate; Arm M: patients treated with methotrexate only.

clinically significant HAQ increase $(\geq 0.22)$ during the first 28 weeks was 5 of 16 patients in the AM arm and 7 of 15 patients in the $\mathrm{M}$ arm $(\mathrm{p}=0.4)$.

The median (IQR) SvH score at baseline was 22.5 (11.352.5) for patients in the AM arm and 42.5 (22-95.3) for patients in the $\mathrm{M}$ arm. One year after randomisation, the SvH score was $25(13.8-51.8)$ and 35.5 (18.3-70.8) for patients in the $\mathrm{AM}$ and $\mathrm{M}$ arms, respectively (three patients in arm $\mathrm{M}$ had no radiological data at year 1). No statistically significant differences were observed in patients in the two arms from baseline to year 1 .

A total of nine patients entered the rescue arm during the first 28 weeks. Remission was restored in eight patients within 12 weeks (the remaining patient achieved remission at the final observational follow-up visit $($ DAS28=2.5) $)$.

\section{Safety data}

The incidence of adverse events was similar in both treatment arms, with $88 \%$ of patients in the AM arm and $100 \%$ of the patients in the $\mathrm{M}$ arm reporting at least one adverse event. No adverse events were reported to cause discontinuation of study treatment or death. One serious adverse event (femur fracture) was reported by a patient in the AM arm and three serious adverse events (malignant melanoma, chest pain and pleuritis) were reported in three patients in the MTX arm. 


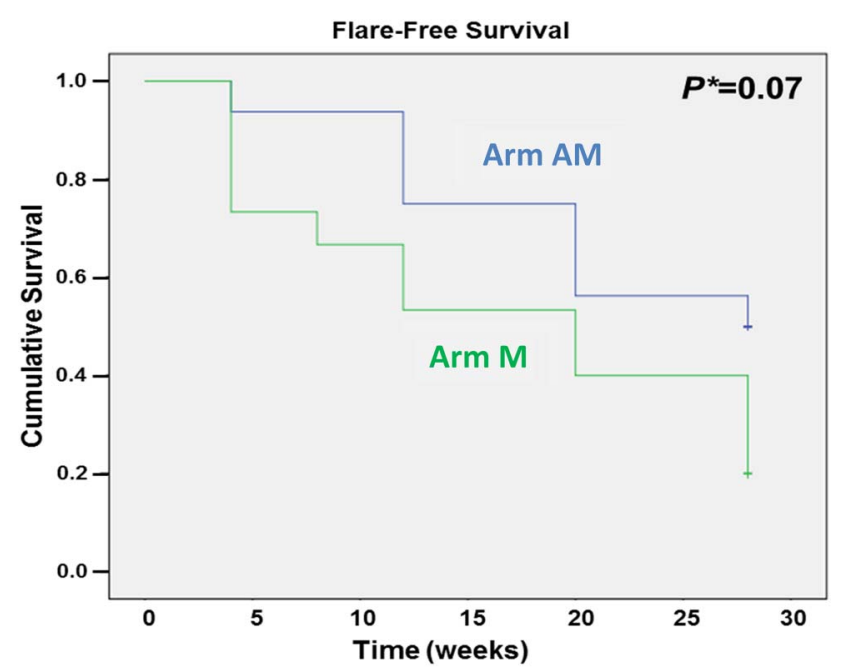

Figure 4 Flare-free survival. Arm AM: patients treated with adalimumab and methotrexate; Arm M: patients treated with methotrexate only.

\section{DISCUSSION}

In this randomised, open-label pilot study, patients with established RA who were in stable remission under treatment with ADA and MTX rarely maintained remission after discontinuation of the biological agent. This result is in agreement with previous discontinuation trials, such as the ATTRACT trial (4).

Several important points need to be considered when interpreting these results. First, patients enrolled in this study had long-standing disease (patients had median disease duration of 8 years). The results should therefore be applicable to patients with more established disease. Similar results were found in other discontinuation studies. In the RRR trial, the mean disease duration was 6 years. In that study, $43 \%$ of patients fulfilled the criteria for clinical remission (DAS28 <2.6) 1 year after discontinuation of infliximab. ${ }^{6}$ In the DOSERA trial, most patients $(87 \%)$ with established RA (median disease duration of 13.6 years) flared after discontinuation of etanercept. ${ }^{11}$ In the HONOR study, $48 \%$ of patients remained in remission after withdrawal of ADA. Patients in this study had mean disease duration of 9 years. ${ }^{9}$ In contrast to the above studies, the possibility of discontinuing the biological agent while maintaining remission seems to be greater in early disease, as shown in other trials. $^{78}$

A second important point is the definition of disease flare. It is obvious from figures $2 \mathrm{~A}-\mathrm{C}$ that the way in which disease flare is defined can greatly influences the results. When a liberal definition of disease flare was used (a single DAS28 $\geq 2.6$ ), the difference between the $\mathrm{AM}$ and the $\mathrm{M}$ arms was no longer significant, since as many as half of the patients continuing ADA therapy experienced a disease flare. This was not unexpected, as DAS28 can vary normally and can be slightly increased even in the absence of a true clinical flare. When different definitions of disease deterioration were used, for example, a combination of DAS28 and $\triangle \mathrm{DAS} 28$ with a specified minimum increase in their value, the difference between the two arms became more obvious. This criterion might be more clinically meaningful. An important lesson from this study is therefore the importance of the choice of the definition of disease flare for future studies. When the ACR/EULAR criteria were applied, few patients remained in remission and no difference between groups could be detected. However, the Boolean definition of remission is much more stringent

Table 2 Baseline disease characteristics for patients who experienced disease flare and those who did not during the first 28 weeks (total and in the two treatment arms)

\begin{tabular}{|c|c|c|c|c|c|c|}
\hline & \multicolumn{2}{|l|}{ AM Arm } & \multicolumn{2}{|l|}{ M Arm } & \multicolumn{2}{|l|}{ All patients } \\
\hline & Flare $(n=8)$ & No flare $(n=8)$ & Flare $(n=12)$ & No flare $(n=3)$ & Flare $(n=20)$ & No flare $(n=11)$ \\
\hline Age (years, median (IQR)) & $51.5(35-60.5)$ & $59.5(53-62.8)$ & $63.5(59.5-66)$ & $65(33-65)$ & $61.5(53.8-65.5)$ & $61(52-65)$ \\
\hline $\begin{array}{l}\text { Disease duration } \\
\text { (years, median (IQR)) }\end{array}$ & $11.2(7.0-22.3)$ & $4.8(3.0-8.2)$ & $10.9(5.2-21.9)$ & $5.5(4.8-14.6)$ & $10.9(5.7-21.9)$ & $5.2(3.7-8.2)$ \\
\hline $\begin{array}{l}\text { Time from RA diagnosis to } \\
\text { ADA (years, median (IQR)) }\end{array}$ & $8.5(3.4-20.7)$ & $2.5(0.5-3.3)$ & $7.6(4.1-16.2)$ & $4.8(3.8-10.3)$ & $8.5(4.1-16.3)$ & $3(1-4.8)$ \\
\hline $\begin{array}{l}\text { Time on ADA treatment } \\
\text { (years, median (IQR)) }\end{array}$ & $2.0(1.2-4.1)$ & $2.4(0.7-4.7)$ & $3.8(1.5-4.3)$ & $1.0(0.7-1.0)$ & $3.0(1.4-4.2)$ & $2.3(0.7-4.3)$ \\
\hline DAS28 & $2.4(2.1-2.5)$ & $1.8(1.3-2.3)$ & $1.7(1.5-2.5)$ & $1.7(0.5-1.8)$ & $2.3(1.7-2.5)$ & $1.7(1.3-1.9)$ \\
\hline SJC & $0(0-1.75)$ & $0(0-0)$ & $0(0-1)$ & $0(0-0)$ & $0(0-1)$ & $0(0-0)$ \\
\hline TJC & $0.5(0-2)$ & $0.5(0-1.8)$ & $0(0-1)$ & $0(0-0)$ & $0(0-1.8)$ & $0(0-1)$ \\
\hline ESR $(\mathrm{mm} \mathrm{Hg})$ & $9(5.8-15.5)$ & $6(4.5-10.5)$ & $8(5-10)$ & $10(2-10)$ & $8(5-11)$ & $6(4-10)$ \\
\hline $\mathrm{CRP}(\mathrm{mg} / \mathrm{L})$ & $3(1.1-5.0)$ & $4.5(0.6-7.0)$ & $4.5(2.5-6.6)$ & $2.9(1.4-5)$ & $4(1.6-5.2)$ & $2.9(1-7)$ \\
\hline $\mathrm{GH}$ & $10(8-20.5)$ & $6(1.5-7.8)$ & $5.5(0.5-10)$ & $5(1-13)$ & $8(4.3-15.3)$ & $6(1-8)$ \\
\hline $\mathrm{HAQ}$ & $0.3(0-0.9)$ & $0.13(0-0.7)$ & $0.4(0.1-0.7)$ & $0.4(0.3-0.5)$ & $0.4(0-0.7)$ & $0.3(0-0.5)$ \\
\hline MTX dose (mg) & $20(15-20)$ & $20(15-20)$ & $18.75(10.6-20)$ & $20(10-20)$ & $20(13.1-20)$ & $20(15-20)$ \\
\hline
\end{tabular}

Values in bold differ significantly between the flare and no flare groups $(p<0.05)$.

ADA, adalimumab; CRP, C reactive protein; DAS28, Disease Activity Score based on 28 joints; ESR, erythrocyte sedimentation rate; GH, global health assessment (patient visual analogue scale); HAQ, Health Assessment Questionnaire; MTX, methotrexate; RA, rheumatoid arthritis; SJC, swollen joint count; TJC, tender joint count. 
and this study, in line with other studies, suggests that DAS28 defined 'remission' might comprise many patients with residual disease activity who are rightly captured as not being in remission by ACR-EULAR remission criteria. ${ }^{22}$

At the end of 28 weeks, the majority of patients in the MTX monotherapy arm experienced disease flare, but three patients $(20 \%)$ had a sustained remission after ADA discontinuation. At the extension visits (weeks 104156), three patients originally allocated to ADA discontinuation had not yet restarted ADA, and of these patients, two were in remission.

This suggests that anti-TNF discontinuation might be feasible even in patients with established RA, but only for a small group of patients. Identification of these patients is, of course, of interest. As shown in table 2, there was a tendency for shorter time from disease onset to ADA initiation in the subgroup of patients who did not experience disease flare, suggesting that earlier initiation of biological DMARDs might increase the chance of retaining remission after discontinuation of the biological agent. However, the number of patients was not large enough to allow any adjusted regression analysis or to draw any firm conclusions about possible prognostic factors of remaining in remission after discontinuation of ADA.

Last but not least, all patients experiencing disease flare who restarted treatment with ADA achieved DAS28-defined remission without any unexpected safety signals. There was no progression of joint damage or deterioration of physical function in either group.

The study has several limitations, including the small number of patients, the open-label design and the issues related to the definition of disease flare. The small number of patients was mainly due to a high number of patients found to be non-eligible after screening. One-third of the patients who were screened for the study were not willing to stop ADA; $16 \%$ were not in stable remission; $12 \%$ were on ADA monotherapy or had a lower dose of MTX than $10 \mathrm{mg} /$ week; and $5 \%$ had a dose of ADA other than $40 \mathrm{mg}$ every other week. Although these were the reasons for the small number of enrolled patients, they were also interesting observations that reflect real-life. A 'nocebo' effect (the reverse of the placebo effect, where the patient's expectation of getting worse causes an actual worsening) might partly contribute to the higher flare rate in the ADA discontinuation group. Some differences in baseline characteristics, such as the longer disease duration, the higher percentage of anticyclic citrullinated peptide-positive patients, the longer duration from diagnosis to ADA initiation in the $\mathrm{M}$ arm compared to the AM arm, could account partly for lower sustainability and more flares in the $\mathrm{M}$ arm. The rescue of 'M' flare patients also biases the patients to better remission rates. The duration of remission (at least 6 months from randomisation/baseline) might be too short, and longer remission duration might be needed before trying to discontinue ADA. This time duration was, however, the same for the two groups. Conversely, the study has some important strengths, such as the randomised, controlled nature of the trial, the homogeneous population of patients and the relevance to the payer perspective on clinical practice.

\section{CONCLUSIONS}

In this pilot study, remission was rarely maintained in patients with RA who discontinued ADA. Compared with patients who continued combination therapy, the proportion of patients with sustained remission in the discontinuation group was significantly lower for the primary end point and most secondary end points. However, discontinuation of ADA may be feasible in a minority of patients with established RA in stable clinical remission on ADA plus MTX.

\section{Author affiliations}

${ }^{1}$ Unit for Clinical Therapy Research, Inflammatory Diseases (ClinTRID), Karolinska Institute, Stockholm, Sweden

${ }^{2}$ Rheumatology, Department of Clinical Sciences, Malmö, Lund University, Malmö, Sweden

${ }^{3}$ Capio Movement, Reumatologi, Halmstad, Sweden

${ }^{4}$ Rheumatology Department, Uppsala University, Uppsala, Sweden

${ }^{5}$ Rheumatology, Department Clinical Sciences Lund, Lund University, Skåne University Hospital, Lund, Sweden

${ }^{6}$ Rheumatology Department, Karolinska University Hospital, Stockholm, Sweden

${ }^{7}$ Rheumatology Department, University Hospital, Linköping, Sweden ${ }^{8}$ Section of Rheumatology, Department of Medicine, Helsingborg Hospital, Helsingborg, Sweden

${ }^{9}$ Rheumatology, Department of Clinical Sciences, Lund University, Helsingborg, Sweden

${ }^{10} \mathrm{AbbVie} A B$, Solna, Stockholm, Sweden

Acknowledgements The authors would like to thank all the investigators, research nurses and patients who participated in this study.

Funding This trial was funded by AbbVie.

Competing interests $\mathrm{KC}$ has received consultancy fees and/or speaker honoraria from Pfizer and Roche. CT has received consultancy fees and/or speaker honoraria from AbbVie, Bristol-Myers Squibb, Janssen, MSD, Pfizer, Roche, Novartis and UCB, and has received unrestricted research grants from AbbVie, Pfizer and Roche. KF has received consultancy fees and/or speaker honoraria from AbbVie, Bristol-Myers Squibb, MSD and Pfizer. RvV has received research support and/or honoraria from AbbVie, Biotest, BMS, GSK, Lilly, Merck, Pfizer, Roche, UCB and Vertex. MH is an employee of AbbVie.

Patient consent Obtained.

Ethics approval Stockholm's county ethics committee.

Provenance and peer review Not commissioned; externally peer reviewed.

Data sharing statement No additional data are available.

Open Access This is an Open Access article distributed in accordance with the Creative Commons Attribution Non Commercial (CC BY-NC 4.0) license, which permits others to distribute, remix, adapt, build upon this work noncommercially, and license their derivative works on different terms, provided the original work is properly cited and the use is non-commercial. See: http:// creativecommons.org/licenses/by-nc/4.0/ 


\section{REFERENCES}

1. Tracey D, Klareskog L, Sasso EH, et al. Tumor necrosis factor antagonist mechanisms of action: a comprehensive review. Pharmacol Ther 2008;117:244-79.

2. Breedveld FC, Weisman MH, Kavanaugh AF, et al. The PREMIER study: a multicenter, randomized, double-blind clinical trial of combination therapy with adalimumab plus methotrexate versus methotrexate alone or adalimumab alone in patients with early, aggressive rheumatoid arthritis who had not had previous methotrexate treatment. Arthritis Rheum 2006;54:26-37.

3. Weinblatt ME, Keystone EC, Furst DE, et al. Adalimumab, a fully human anti-tumor necrosis factor alpha monoclonal antibody, for the treatment of rheumatoid arthritis in patients taking concomitant methotrexate: the ARMADA trial. Arthritis Rheum 2003;48:35-45.

4. Lipsky PE, van der Heijde DM, St Clair EW, et al, Anti-Tumor Necrosis Factor Trial in Rheumatoid Arthritis with Concomitant Therapy Study Group. Infliximab and methotrexate in the treatment of rheumatoid arthritis. Anti-Tumor Necrosis Factor Trial in Rheumatoid Arthritis with Concomitant Therapy Study Group. N Engl J Med 2000;343:1594-602.

5. Quinn MA, Conaghan PG, O'Connor PJ, et al. Very early treatment with infliximab in addition to methotrexate in early, poor-prognosis rheumatoid arthritis reduces magnetic resonance imaging evidence of synovitis and damage, with sustained benefit after infliximab withdrawal: results from a twelve-month randomized, double-blind, placebo-controlled trial. Arthritis Rheum 2005;52:27-35.

6. Tanaka Y, Takeuchi T, Mimori T, et al. Discontinuation of infliximab after attaining low disease activity in patients with rheumatoid arthritis: RRR (remission induction by Remicade in RA) study. Ann Rheum Dis 2010;69:1286-91.

7. van der Kooij SM, le Cessie S, Goekoop-Ruiterman YP, et al. Clinical and radiological efficacy of initial vs delayed treatment with infliximab plus methotrexate in patients with early rheumatoid arthritis. Ann Rheum Dis 2009;68:1153-8.

8. Smolen JS, Emery P, Fleischmann R, et al. Adjustment of therapy in rheumatoid arthritis on the basis of achievement of stable low disease activity with adalimumab plus methotrexate or methotrexate alone: the randomised controlled OPTIMA trial. Lancet 2014;383:321-32.

9. Tanaka Y, Hirata S, Kubo S, et al. Discontinuation of adalimumab after achieving remission in patients with established rheumatoid arthritis: 1-year outcome of the HONOR study. Ann Rheum Di 2015;74:389-95.

10. Emery $\mathrm{P}$, Hammoudeh $\mathrm{M}$, FitzGerald $\mathrm{O}$, et al. Sustained remission with etanercept tapering in early rheumatoid arthritis. $N$ Engl J Med 2014;371:1781-92.
11. van Vollenhoven RF, Østergaard M, Leirisalo-Repo M, et al. Full dose, reduced dose or discontinuation of etanercept in rheumatoid arthritis. Ann Rheum Dis 2016;75:52-8.

12. Smolen JS, Nash $P$, Durez $P$, et al. Maintenance, reduction, or withdrawal of etanercept after treatment with etanercept and methotrexate in patients with moderate rheumatoid arthritis (PRESERVE): a randomised controlled trial. Lancet 2013;381:918-29.

13. Smolen JS, Emery P, Ferraccioli GF, et al. Certolizumab pegol in rheumatoid arthritis patients with low to moderate activity: the CERTAIN double-blind, randomised, placebo-controlled trial. Ann Rheum Dis 2015;74:843-50.

14. Arnett FC, Edworthy SM, Bloch DA, et al. The American Rheumatism Association 1987 revised criteria for the classification of rheumatoid arthritis. Arthritis Rheum 1988;31:315-24.

15. Prevoo ML, van 't Hof MA, Kuper $\mathrm{HH}$, et al. Modified disease activity scores that include twenty-eight-joint counts. Development and validation in a prospective longitudinal study of patients with rheumatoid arthritis. Arthritis Rheum 1995;38:44-8.

16. Ekdahl C, Eberhardt K, Andersson SI, et al. Assessing disability in patients with rheumatoid arthritis. Use of a Swedish version of the Stanford Health Assessment Questionnaire. Scand J Rheumatol 1988;17:263-71.

17. Felson DT, Smolen JS, Wells G, et al. American College of Rheumatology/European League Against Rheumatism provisional definition of remission in rheumatoid arthritis for clinical trials. Arthritis Rheum 2011;63:573-86.

18. Felson DT, Smolen JS, Wells G, et al. American College of Rheumatology/European League against Rheumatism provisional definition of remission in rheumatoid arthritis for clinical trials. Ann Rheum Dis 2011;70:404-13.

19. Wolfe F. Comparative usefulness of $\mathrm{C}$-reactive protein and erythrocyte sedimentation rate in patients with rheumatoid arthritis. $J$ Rheumatol 1997;24:1477-85.

20. van der Heijde DM. Plain X-rays in rheumatoid arthritis: overview of scoring methods, their reliability and applicability. Baillieres Clin Rheumatol 1996;10:435-53.

21. van der Heijde D. How to read radiographs according to the Sharp/ van der Heijde method. J Rheumatol 1999;26:743-5.

22. Smolen JS, Wollenhaupt J, Gomez-Reino JJ, et al. Attainment and characteristics of clinical remission according to the new ACR-EULAR criteria in abatacept-treated patients with early rheumatoid arthritis: new analyses from the Abatacept study to Gauge Remission and joint damage progression in methotrexate (MTX)-naive patients with Early Erosive rheumatoid arthritis (AGREE). Arthritis Res Ther. 2015;17:157. 